\title{
Stress state of a polygonal plate having a central circular hole with two linear cracks in the small physical non-linear condition
}

\author{
Etimad Bayramoglu Eyvazov ${ }^{1}$ and Mecit Öge ${ }^{2}$ \\ ${ }^{1}$ Karabuk University, Faculty of Engineering, Mechanical Engineering Department, Karabuk, Turkey \\ ${ }^{2}$ Bartin University, Faculty of Engineering, Mechanical Engineering Department, 74100. Bartin, Turkey \\ Correspondence: Mecit Öge (mecitoge@yahoo.com)
}

Received: 16 May 2019 - Accepted: 7 October 2019 - Published: 18 November 2019

\begin{abstract}
In this study, the stress state of a polygonal plate having a central circular hole with two linear cracks was considered. External forces were applied to the contours of polygonal plate and internal forces were applied to the contours of the circular hole. Conformal mapping function, initially developed by Kuliyev, was used to the make stress analysis of the polygonal plate in physical non-linear statement. As a result, stress concentrations were determined by using conformal mapping function at the end points of cracks. Critical loads where fracture began were defined for different loads. Analytical results of solutions were compared and found to be in agreement with the numerical results in the literature.
\end{abstract}

\section{Introduction}

In recent years, solution of physical non-linear problems in the fields of physics and engineering that cannot be solved through linearization of assumptions and principal equations (such as stress-strain relationship), has necessitated making improvements in classical elasticity theory, which in turn has led to the development of non-linear elasticity theory.

All assumptions and resulting errors mentioned in elasticity theory up to date have shown that, there is insufficient information on the new variants of non-linear elasticity theory. In classic elasticity theory, linearization is mainly assumed to be in two directions. The first one is the geometry of a deformable body and the other is physical properties of material (relationship of stress-strain, i.e. the Hooke's law).

Since classical elasticity theory cannot be applied for nonlinear materials, Muskhelishvili (1962) developed a method to solve the stress states of non-linear materials, in which complex variable functions are used to make stress analysis.

Stress concentration around a hole in an infinite plate subjected to a uniform load was investigated by Batista (2011) using Muskhelishvili's (1962) conformal mapping complex variable method. Pan et al. (2013) performed the stress analysis of a finite plate having a rectangular hole subjected to uniaxial tension using a modified form of Muskhelishvili's (1962) conformal mapping function. Sharma (2012) examined the stress distribution around polygonal holes in an infinite plate subjected to arbitrary biaxial in-plane loadings at infinity also by using Muskhelishvili's (1962) complex variable method. In his study, plane stress finite models were prepared in ANSYS and the results were compared with those of the complex variable method (Sharma, 2012). Sharma (2015) obtained a general solution in hypocycloidal hole with cusps in infinite anisotropic plate in order to calculate the stress intensity factors by using Muskhelishvili's (1962) complex variable method. Kuliyev (2003), on the other hand, investigated the stress-strain state of a polygonal plate weakened by a central elliptic hole with two linear cracks by using modified conformal mapping function. Kuliyev (2010) also obtained the solution for critical angular velocity in polygonal plate weakened by elliptic holes using a modified version of conformal mapping function.

In this paper, stress components of a polygonal plate having central circular hole with two linear cracks were obtained. Conformal mapping function which was developed by the author (Kuliyev, 1991, 2017) was used to determine the stress intensity factors for complex geometries. In this work, a complex geometry with additional two linear cracks 
is solved for the first time using Kuliyev's mapping function, and the results were compared with the literature findings which proved to be in good agreement.

\section{Problem statement and solution methods}

Physical law of deformability, i.e., relationship of stressstrain components is explained in two ways:

1. General functional relationship of stress and strain (between two different power symmetric tensor components) (Kauderer, 1961).

2. Inclusion of deformation energy function into elasticity potential (Globenko, 1974).

As mentioned above, determination of real physical relationships between stress-strain components and solution of some complex problems in engineering constitute the essence of non-linear elasticity theory.

As reported in the literature (Kuliyev, 1991, 2001; Amenzade, 1976; Berezhnitskii et al., 1919; Sherman, 1947, 1951) Airy Stress Function $F^{(n)}$ is determined by two analytic functions, namely $\varphi(z)$ and $\psi(z)$ in linear elasticity theory (plane problems)

$F^{(0)}=\operatorname{Re}\left[\bar{z} \cdot \varphi(z)+\int \psi(z) \mathrm{d} z\right]$

where, Re shows the real part of the expression in bracket.

The functions of elasticity theory named as $\varphi(z)$ and $\psi(z)$ in finite or infinite form of bodies (plate, cylinder, prism and etc.) and connected domains are taken as follows (Kuliyev, 1991, 2001; Amenzade, 1976; Berezhnitskii et al., 1919; Sherman, 1947, 1951).

\subsection{For finite doubly - connected domains}

The regular functions $\varphi(z)$ and $\psi(z)$ are as follows (Kuliyev, 1991, 2001; Amenzade, 1976; Sherman, 1947, 1951).

$$
\begin{aligned}
& \varphi(z)=\sum_{k=1}^{\infty} \alpha_{k} \cdot \xi_{1}^{-k}+\sum_{k=0}^{\infty} A_{k}\left(\frac{z}{A}\right)^{k} \\
& \psi(z)=\sum_{k=1}^{\infty} \beta_{k} \cdot \xi_{1}^{-k}+\sum_{k=0}^{\infty} B_{k}\left(\frac{z}{A}\right)^{k}
\end{aligned}
$$

where $\xi_{1}$ is the function of $\xi=\chi(z)$, inverse function $(z=$ $\left.\omega\left(\xi_{1}\right)\right)$ of which conforms $L_{1}$ inner contour around the unit circle in Fig. 1 (Kuliyev, 1991, 2001).

The coefficients $A_{k}$ and $B_{k}$ of analytic functions are as follows:

$$
\begin{aligned}
& A_{k}=\sum_{n=k}^{\infty} * a_{n}^{(k)} \cdot a_{\frac{n-k}{q}}^{(n)} \\
& B_{k}=\sum_{n=k}^{\infty} b_{n} \cdot a_{\frac{n-k}{q}}^{(n)}
\end{aligned}
$$

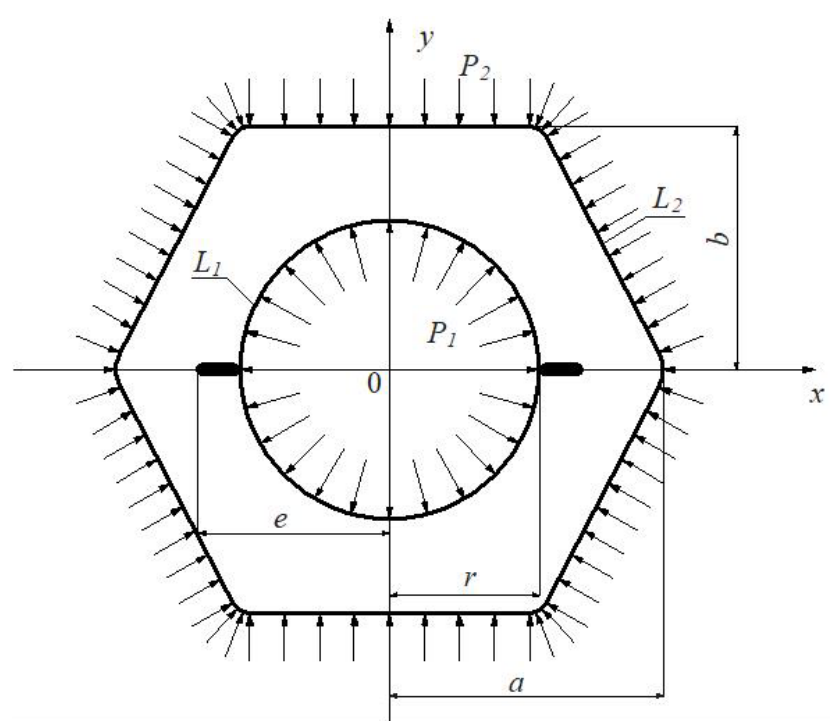

Figure 1. A hexagonal cross-section under interior and exterior pressure.

The coefficient $a_{n}^{(k)}$ in Eq. (3) is found for total contour and given in table form in (Kuliyev, 2017). The symbol * indicates the indices in series Eq. (3) with increment $q$. We assume that contours $L_{1}$ and $L_{2}$ are concentric in doubly connected domains.

Here, $F^{(0)}$ is the Airy stress function in zero approximation. The function $F^{(0)}$ is given by the known formula (the plate is under the influence of internal $p_{1}$ and external pressure $p_{2}$ equi-distributed hydrostatic pressure in Fig. 1) for polygonal doubly connected plates (Kuliyev, 1991, 2001; Muskhelishvili, 1962; Amenzade, 1976; Berezhnitskii et al., 1919).

In the considered case, the boundary conditions are as follows in zero approximation:

- on the inner contour $L_{1}$ (with $r$ radius circle and two linear cracks)

$$
\left.\sigma_{\rho}^{(0)}\right|_{\rho=t_{1}}=-P_{1}
$$

on the outer contour $L_{2}$ (equilateral polygon)

$$
\left.\sigma_{\rho}^{(0)}\right|_{\rho=t_{2}}=-P_{2}
$$

For the next approximations (the first and the second), the boundary conditions are as follows:

$\left.\sigma_{\rho}^{(1)}\right|_{\rho=t_{1}}=0,\left.\sigma_{\rho}^{(1)}\right|_{\rho=t_{2}}=0$

In Eqs. (4), (5), (6), $t_{1}$ and $t_{2}$ are the affixes of the points of the contours $L_{j}(j=1 ; 2)$, respectively. The function $F^{(1)}$ is found from the solution of Eq. (1).

$$
\Delta \Delta F^{(2)}+L_{2}\left[F^{(0)}, F^{(1)}\right]=0
$$


The solution of Eq. (7) is found from the sum of general solution $\Delta \Delta F^{(1)}=0$ of the first differential equation $\left(F_{\text {gen. }}^{(1)}\right)$ and the special solution $\left(F_{\text {spec. }}^{(1)}\right)$ of the mentioned differential equation.

$F^{(1)}=F_{\text {gen. }}^{(1)}+F_{\text {spec. }}^{(1)}$

It is known that the general solution of the homogeneous differential equation $\Delta \Delta F^{(1)}=0$ is taken in the following way (in the general case):

$F_{\text {gen. }}^{(1)}=c_{1} \cdot \ln \rho+c_{2} \cdot \rho^{2}$

Note that, if we pass to polar $(\rho, \theta)$ coordinates, we get the following differential equation for the function $F^{(1)}$ (Kuliyev, 2017).

$\Delta \Delta F^{(1)}=\sum_{k=1}^{\infty} \rho^{-k} \cdot V_{1}(k)+\sum_{k=0}^{\infty} \rho^{k} \cdot V_{2}(k)$

In this equation, the quantities $V_{1}(k)$ and $V_{2}(k)$ are algebraic sums of the same power terms of the variable $p$ (for the fixed $\theta$ angle). The special solution $F_{\text {spec. }}^{(1)}$ is found from fourfold integration of Eq. (10) in the following way (Kuliyev, 1991, 2001):

$$
\begin{aligned}
F_{\text {spec. }}^{(1)} & =\sum_{k=0}^{\infty} \rho^{-k+4} \cdot \alpha \cdot V_{1}(k) \\
& +\sum_{k=0}^{\infty} \beta \cdot \rho^{k+4} \cdot V_{2}(k)
\end{aligned}
$$

where

$$
\begin{aligned}
& \alpha=\left[(4-k)^{2} \cdot(2-k)^{2}+\alpha_{1}+\alpha_{2}\right]^{-1} \\
& \beta=\left[(k+4)^{2} \cdot(2+k)^{2}+\beta_{1}+\beta_{2}\right]^{-1}
\end{aligned}
$$

The unknown coefficients $c_{1}$ and $c_{2}$ in Eq. (9) are found from the boundary conditions of the considered problem (stressstrain state of a doubly-connected polygonal plate subjected to equi-distributed internal pressure $p_{1}$ and external pressure $p_{2}$ ) (Kuliyev, 1991, 2001).

If on the $L_{1}$ inner contour

$\rho=t_{1}$ then $\sigma_{\rho}^{(1)}=0$

If on the $L_{2}$ external contour

$\rho=t_{2}=A$ then $\sigma_{\rho}^{(1)}=0$

After finding the expression $F(\rho, \theta, \lambda t)=F^{(0)}(\rho, \theta)+$ $\lambda F^{(1)}(\rho, \theta)+\lambda^{2} \cdot F^{(2)}(\rho, \theta)+\ldots$ for the stress components $\sigma_{\rho}$ and $\sigma_{\theta}$ we get the following expressions in the form of the following series:

$$
\begin{aligned}
\sigma_{\rho} & =\sigma_{\rho}^{(0)}+\lambda \cdot \sigma_{\rho}^{(1)}+\lambda^{2} \cdot \sigma_{\rho}^{(2)}+\ldots \\
\sigma_{\theta} & =\sigma_{\theta}^{(0)}+\lambda \cdot \sigma_{\theta}^{(1)}+\lambda^{2} \cdot \sigma_{\theta}^{(2)}+\ldots \\
\tau_{\rho \theta} & =\tau_{\rho \theta}^{(0)}+\lambda \cdot \tau_{\rho \theta}^{(1)}+\lambda^{2} \cdot \tau_{\rho \theta}^{(2)}+\ldots
\end{aligned}
$$

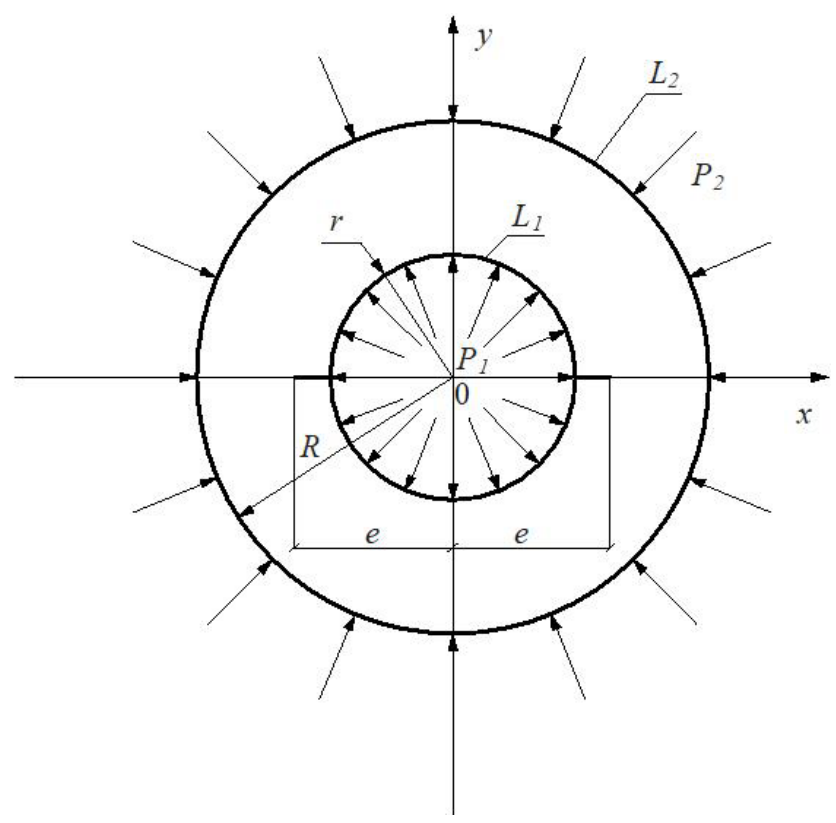

Figure 2. Hollow round cylinder under interior and exterior pressure.

The obtained general solution is demonstrated by the numerical examples.

\subsection{Numerical Results}

\subsubsection{Stress state of a cylinder made of small physical nonlinear material and possessing an annular hole and two linear cracks}

In linear elasticity theory, this problem was solved completely (within Hooke's law). Therefore, here we give only the results obtained in Kuliyev $(1991,2001)$.

The ratio of the main measures of the cross section is given in two variants (Fig. 2):

- Variant I: $r / R=0.5 ; e=0.6 R ; l=0.1 R$

- Variant II: $r / R=0.7 ; e=0.8 R ; l=0.1 R$.

The stresses $\sigma_{\rho}^{(0)}$ and $\sigma_{\theta}^{(0)}$ found from characteristic points of the section (as tangential stresses $\tau_{\rho \theta}$ are too small, accepted $\tau_{\rho \theta} \approx 0$ ) are given in Table 1 . Stress intensities and critical stress values are given in Table 2 .

At the most critical point of the section (at the end points of the cracks: $z= \pm e$ ), we get the following values of $10 K_{1} / p_{1} \sqrt{l}$ for the stress intensity factor $K_{1}$ :

- 7.00 for variant I and

- 11.68 for variant II.

The following estimates are found for the critical value of the internal pressure $p_{1}=p$ (i.e. fracture of the body-minimal stress for elongation of cracks): 


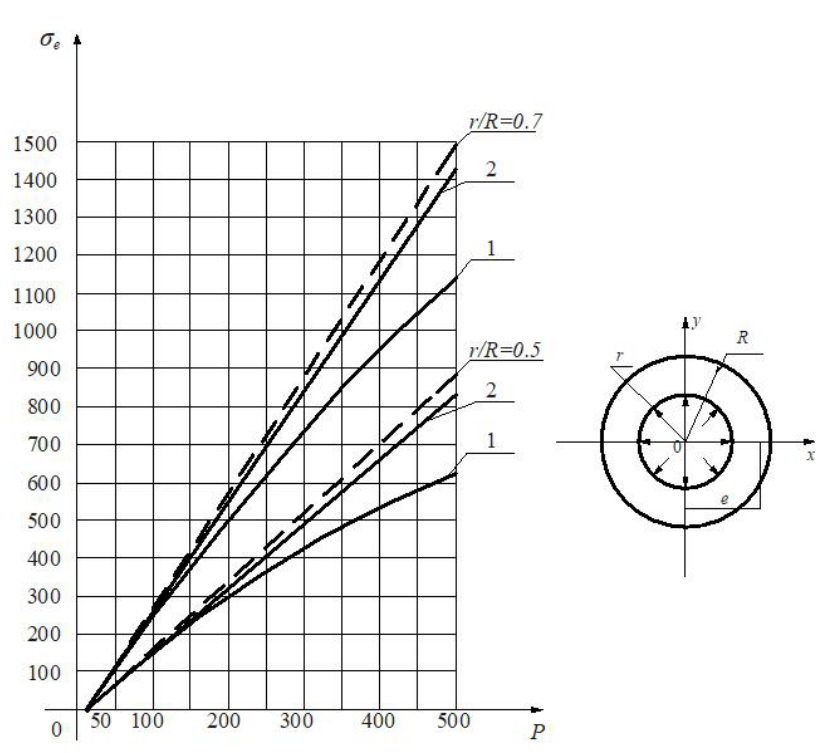

Figure 3. Hollow cylinder under interior pressure. The dotted line indicates a linear problem, the solid line a non-linear problem. (1: pure copper; 2: open heath steel).

- For variant I: $P_{\mathrm{cr}}=1,46\left[\sigma_{b}\right]$

- For variant II: $P_{\mathrm{cr}}=0,883\left[\sigma_{b}\right]$ (Table 2)

where technical ultimate stress $\left[\sigma_{b}\right]$ (maximum stress of the material) is known from references (Kuliyev, 1991, 2001).

After finding the functions $\varphi(z)$ and $\psi(z)$, within the linear elasticity theory (in zero approximation), the stress function $F^{(0)}(x, y)=F^{(0)}(\rho, \theta)$ is found by Eq. (1). Then $F^{(1)}(\rho, \theta)$ is found from Eq. (10) of the stress function in the first approximation. According to the found expression of the stress function $F^{(1)}(\rho, \theta)$, the stress $\sigma_{\theta}$ found from the characteristic points of the section by means of Eq. (14) is given in and Fig. 3.

\subsubsection{Stress state of a hexagonal plate with annular hole and two linear cracks in physical non-linear statement}

The solution of this problem in linear elasticity theory is known from references (Kuliyev, 1991, 2001). The stresses $\left(\sigma_{\theta}^{(0)}\right)$ found at any point of a hexagonal plate are calculated for different variants (for different values of the ratio of cross section measures) and given in the table. The stress intensity factor $\left(K_{1}\right)$ and critical value $P_{\mathrm{cr}}$ of internal pressure are found and given for each variant at the end of Tables 3 and 4 according to the stresses found at the end points of the linear cracks. The known expression of the function is taken into account in differential Eq. (10) obtained for the solution of physical non-linear problem and accordingly, the stress for different values of internal pressure $P_{\text {cr }}$ at the most critical points $F^{(0)}(x, y)=F^{(0)}(\rho, \theta)$ of the section are found and given in table form.

The following expressions were considered for the stresses at the tips of the cracks.

- Variant I: $r / A=0.5 ; e / A=0.6 ; m=0.04 ; b=1,922$;

$$
\sigma_{\theta} / p=\sigma_{\theta / p}^{(0)}+\alpha \sigma_{\theta}^{(1)} / p=1.68-9.2 \cdot \alpha \cdot p^{2}
$$

- Variant II: $r / A=0.7 ; e / A=0.8 ; m=0.04 ; b=1.37 r$

$\sigma_{\theta} / p=\sigma_{\theta / p}^{(0)}+\alpha \sigma_{\theta}^{(1)} / p=3.21-14.62 \cdot \alpha \cdot p^{2}$

where

$$
\alpha=\lambda=\frac{g_{2} k}{(3 K+G) G^{2}}=0.225 \times 10^{-6} \mathrm{~cm}^{4} \mathrm{~kg}^{-2}
$$

for pure copper.

$$
\alpha=\lambda=0.032 \times 10^{-6} \mathrm{~cm}^{4} \mathrm{~kg}^{-2}
$$

for open-hearth steel.

As indicated by the numerical results of both problems in physical non-linear statement, the maximal values of stresses are smaller than that of the linear statement (peak points of stress diagrams are smoothened) (Fig. 1).

\subsubsection{Stress state of a thick-walled pipe (Lame problem) in small physical non-linear statement}

In classic elasticity theory, the following formula is obtained for the stress function $F^{(0)}$ (Kuliyev, 1991, 2001).

$F^{(0)}(\rho, \theta)=\beta_{1} \cdot \frac{\rho^{2}}{r^{2}}+d_{1} \cdot r \cdot \ln \rho$

When the pipe is under the internal pressure $p_{1}$ and external hydrostatic pressure $p_{2}$, the coefficients $\beta_{1}$ and $d_{1}$ are determined by the following expressions (Kuliyev, 1991, 2001).

$\beta_{1}=\frac{r}{2} \cdot \frac{p_{1} \cdot r^{2}-p_{2} \cdot R^{2}}{R^{2}-r^{2}} ; d_{1}=\frac{r^{2} R^{2} \cdot\left(p_{2}-p_{1}\right)}{\left(R^{2}-r^{2}\right) \cdot r}$

if the Eq. (16) is written in Eq. (15);

$$
\begin{aligned}
F^{(0)}(\rho, \theta) & =\frac{r^{2} R^{2} \cdot\left(p_{2}-p_{1}\right)}{R^{2}-r^{2}} \cdot \ln \rho \\
& +\frac{1}{2} \frac{p_{1} \cdot r^{2}-p_{2} \cdot R^{2}}{R^{2}-r^{2}} \rho^{2}
\end{aligned}
$$

The obtained expression completely coincides with the expressions obtained by Tsurpal (1962). The measures of thickwalled pipes (the ratio of radius $R$ of external circle with radius $r$ of internal circle) were given in two variants (pure copper, aluminum, bronze and open-heath steel were chosen as pipe's materials. The following expressions are obtained for stress concentration on the inner circle for the considered problem (Lame Problem). 
Table 1. Stress coefficient concentrations.

\begin{tabular}{lrrlll}
\hline Variants & $P$ & $\begin{array}{r}\text { Linear } \\
\text { statement }\end{array}$ & $\begin{array}{l}\text { Pure copper } \\
\lambda=0.255 \times 10^{-6}\end{array}$ & $\begin{array}{l}\text { Aluminum Silver } \\
\lambda=0.053 \times 10^{-6}\end{array}$ & $\begin{array}{l}\text { Open heath steel } \\
\lambda=0.032 \times 10^{-6}\end{array}$ \\
\hline $\mathrm{I}$ & 20 & 1.76 & 1.759102 & 1.7598317 & 1.7593984 \\
$r / R=0.5$ & 50 & 1.76 & 1.7549383 & 1.758948 & 1.759365 \\
& 100 & 1.76 & 1.73975 & 1.755792 & 1.75746 \\
$e / R=0.6$ & 200 & 1.76 & 1.6791012 & 1.74317 & 1.74984 \\
& 500 & 1.76 & 1.253825 & 1.654795 & 1.6965 \\
\hline $\mathrm{II}$ & 20 & 2.92 & 2.918793 & 2.419738 & 2.919842 \\
$r / R=0.7$ & 50 & 2.92 & 2.912123 & 2.91836 & 2.91901 \\
& 100 & 2.92 & 2.88848 & 2.91345 & 2.916065 \\
$e / R=0.8$ & 200 & 2.92 & 2.79393 & 2.8938 & 2.9042 \\
& 500 & 2.92 & 2.23205 & 2.75623 & 2.82112 \\
\hline
\end{tabular}

Table 2. Stress intensity (by $10 K_{1} / P \sqrt{l}$ ) and critical value of stress.

\begin{tabular}{lrrr|rr}
\hline Variant & Pressure $P$ & \multicolumn{2}{c}{ Pure copper $\lambda-0.255 \times 10^{-6}$} & \multicolumn{2}{c}{ Open heath steel } \\
\cline { 3 - 6 } & & $K_{1}$ & Critical pressure $\left(P_{\mathrm{cr}}\right)$ & $K_{1}$ & Critical load $P_{\mathrm{cr}}$ \\
\hline $\mathrm{I}$ & 100 & 6.958 & $1.48\left[\sigma_{b}\right]$ & 7.024 & $1.467\left[\sigma_{b}\right]$ \\
$\frac{r}{R}=0.5$ & 200 & 6.726 & $1.535\left[\sigma_{b}\right]$ & 6.999 & $1.473\left[\sigma_{b}\right]$ \\
$\frac{e}{R}=0.6$ & 500 & 5.015 & $2.05\left[\sigma_{b}\right]$ & 6.792 & $1.51\left[\sigma_{b}\right]$ \\
\hline $\mathrm{II}$ & 100 & 11.552 & $0.892\left[\sigma_{b}\right]$ & 11.664 & $0.884\left[\sigma_{b}\right]$ \\
$\frac{r}{R}=0.7$ & 200 & 22.176 & $0.922\left[\sigma_{b}\right]$ & 11.616 & $0.887\left[\sigma_{b}\right]$ \\
$\frac{e}{R}=0.8$ & 500 & 8.928 & $1.155\left[\sigma_{b}\right]$ & 11.284 & $0.914\left[\sigma_{b}\right]$ \\
\hline
\end{tabular}

Table 3. Stress concentrain of a hexogonal plate.

\begin{tabular}{|c|c|c|c|c|c|}
\hline \multirow[t]{2}{*}{ Variants } & \multirow[t]{2}{*}{$P$} & \multirow{2}{*}{$\begin{array}{r}\text { Linear theory } \\
\sigma_{\theta} / p\end{array}$} & \multicolumn{3}{|c|}{ Non linear theory } \\
\hline & & & $\begin{array}{r}\text { Pure copper } \\
\lambda=0.255 \times 10^{-6}\end{array}$ & $\begin{array}{l}\text { Alluminium silver } \\
\lambda=0.053 \times 10^{-6}\end{array}$ & $\begin{array}{l}\text { Open heath steel } \\
\lambda=0.032 \times 10^{-6}\end{array}$ \\
\hline I & 20 & 1.68 & 1.679185 & 1.6659 & 1.6798984 \\
\hline \multirow{2}{*}{$\frac{r}{A}=0.5$} & 50 & 1.68 & 1.67494 & 1.6654 & 1.6793648 \\
\hline & 100 & 1.68 & 1.65975 & 1.6636 & 1.67746 \\
\hline \multirow{2}{*}{$\frac{e}{A}=0.6$} & 200 & 1.68 & 1.59901 & 1.6566 & 1.669835 \\
\hline & 500 & 1.68 & 1.17384 & 1.607 & 1.61648 \\
\hline II & 20 & 3.21 & 3.20874 & -2.66588 & -2.66593 \\
\hline \multirow{2}{*}{$\frac{r}{A}=0.7$} & 50 & 3.21 & 3.20212 & -2.6652 & -2.6655 \\
\hline & 100 & 3.21 & 3.168482 & -2.6515 & -2.6629 \\
\hline \multirow{2}{*}{$\frac{e}{A}=0.8$} & 200 & 3.21 & 3.083928 & -2.654 & -2.6587 \\
\hline & 500 & 3.21 & 2.52205 & -2.591 & -2.2106 \\
\hline
\end{tabular}

- Variant I: $\sigma_{\theta} / p=1.666-4.444 \cdot \lambda \cdot p^{2} ; p_{1}=p$

- Variant II: $\sigma_{\theta} / p=4.55-25.3 \cdot \lambda \cdot p^{2} ; p_{1}=p$

The stresses " $\sigma_{\theta}$ " were calculated for different values of internal pressure $\left(p_{1}\right)(20 \leq p \leq 500)$ by means of the formulas given in graphic form in both variants (Fig. 4). In the graph, the straight line represents the problem in non-linear statement and the dotted line represents the problem in linear statement.
As seen in the tables, a decline at a rate of $0.1 \%$ occurs for the values lower than $100 \mathrm{MPa}$, resulting in a negligible difference between linear and non-linear states. As for the values higher than or equal to $100 \mathrm{MPa}$, the stress difference between linear and nonlinear states is observed to be significantly high. 
Table 4. Stress intensity coefficient $\left(10 K_{1} / \mathbb{I} \sqrt{l}\right)$ and critical value of load $\left(P_{\mathrm{cr}}\right)$ (hexagonal cylinder).

\begin{tabular}{lrrr|rr}
\hline Variant & Pressure $P$ & \multicolumn{2}{c}{ Pure copper $\lambda=0.255 \times 10^{-6}$} & \multicolumn{2}{c}{ Open heath steel } \\
\cline { 3 - 6 } & & $K_{1}$ & Critical pressure $\left(P_{\mathrm{cr}}\right)$ & $K_{1}$ & Critical load $\left(P_{\mathrm{cr}}\right)$ \\
\hline $\mathrm{I}$ & 100 & 6.624 & $1.56\left[\sigma_{b}\right]$ & 6.708 & $1.5386\left[\sigma_{b}\right]$ \\
$\frac{r}{R}=0.5$ & 200 & 6.352 & $1.623\left[\sigma_{b}\right]$ & 6.6792 & $1.543\left[\sigma_{b}\right]$ \\
$\frac{e}{R}=0.6$ & 500 & 4.4 & $2.343\left[\sigma_{b}\right]$ & 6.464 & $1.595\left[\sigma_{b}\right]$ \\
\hline $\mathrm{II}$ & 100 & 12.68 & $0.803\left[\sigma_{b}\right]$ & 12.824 & $0.804\left[\sigma_{b}\right]$ \\
$\frac{r}{R}=0.7$ & 200 & 12.24 & $0.842\left[\sigma_{b}\right]$ & 12.776 & $0.807\left[\sigma_{b}\right]$ \\
$\frac{e}{R}=0.8$ & 500 & 11.92 & $0.865\left[\sigma_{b}\right]$ & 12.44 & $0.829\left[\sigma_{b}\right]$ \\
\hline
\end{tabular}

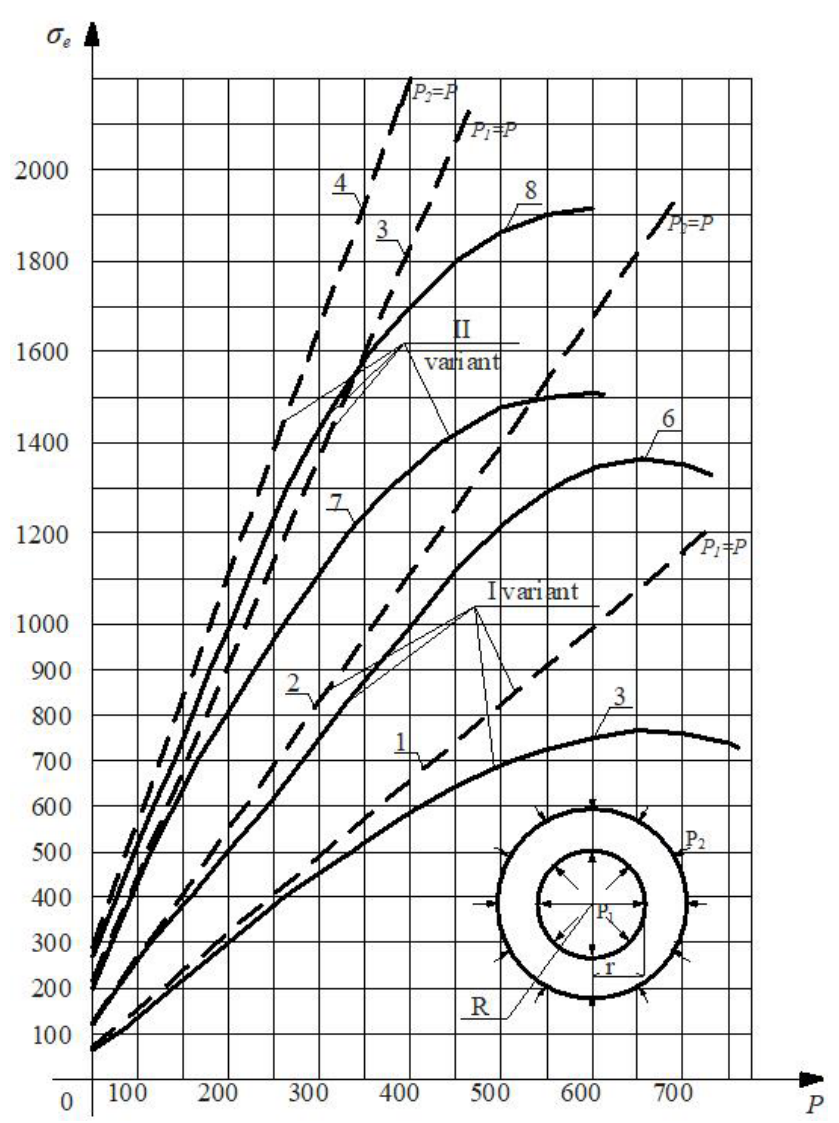

Figure 4. The Lame problem statement.

\section{Conclusion}

In this work, stress concentrations were determined by using conformal mapping function at the end points of cracks. Critical loads where fracture began were defined for different loads. Analytical results of solutions were compared and found to be in agreement with the numerical results in the literature. The obtained numerical calculations show that, as the nonlinearity of the materials increase, the stress distributions at the peak points of the stresses become more regular and physical-nonlinearity of the material smoothens the peaks of stresses by decreasing maximum value of stresses and moderately increasing minimum values. Also, the physical nonlinearity of the material decreases the stress concentration, thus decreasing the maximum stress at the inner points of the cross-section.

Data availability. The underlying research data can be provided upon reasonable request by Etimad Bayramoglu Eyvazov (etimadeyvazov@karabuk.edu.tr). 
Appendix A: Nomenclature

$K \quad$ bulk modulus

$k\left(s_{0}\right) \quad$ mean stress of $s_{0}$ function

$g\left(t_{0}^{2}\right) \quad$ intensity function

$G \quad$ shear modulus

$s_{0} \quad$ expression for the reduced mean stress

E $\quad$ Young modulus

F $\quad$ Airy stress function

$I_{1} \quad$ strain tensor

$L^{(n)} \quad$ linear operator

$\lambda_{i j} \quad$ the coefficients which determine the physical properties of the considered materials

$\tau_{0} \quad$ expression for intensity of the reduced tangential stresses

$v \quad$ Poisson ratio

$\chi \quad$ elongation function

$\Upsilon \quad$ shear strain function

$\varepsilon_{i j} \quad$ strain components

$\delta_{i j} \quad$ the coefficients which determine the physical properties of the considered materials

$\sigma_{i j} \quad$ stress components

$\Delta \quad$ Laplace operator

$\varphi(z), \psi(z) \quad$ analitic functions

$q \quad$ symmetry axis number 
Author contributions. EBE conducted the numerical analyses and wrote the majority of the paper. MÖ supervised the findings, organized and structured the paper.

Competing interests. The authors declare that they have no conflict of interest.

Acknowledgements. The authors gratefully acknowledge the valuable support of Sabir A. Kuliyev, who recently passed away.

Review statement. This paper was edited by Doina Pisla and reviewed by Farshid Khosravi Maleki and one anonymous referee.

\section{References}

Amenzade, Y. A.: Theory of Elasticity, Vysshaya Shkola, Moscow, 1976 (in Russian).

Batista, M.: On the stress concentration around a hole in an infinite plate subject to a uniform load at infinity, Int. J. Mech. Sci., 53, 254-261, https://doi.org/10.1016/j.ijmecsci.2011.01.006, 2011.

Berezhnitskii, L. T., Delyavskii, M. V., and Panasyuk, V. V.: Bending of thin plates with crack type defects, Naukova Dumka, Kiew, 1919 (in Russian).

Globenko, T. M.: Influence of physical nonlinearity of material on stress concentration near square hole, Calc. Meth. Civ. Eng., 1, 39-55, 1974.

Kauderer, G.: Nonlinear mechanics, Inostrannaya Literatura, Moscow, 1961
Kuliyev, S.: Stress state of compound polygonal plate, Mech. Res. Com., 30, 519-530, 2003.

Kuliyev, S.: Uniform rotation of a polygonal plate weakened by two linear crack holes, Mech. Res. Com., 37, 184-190, 2010.

Kuliyev, S. A.: Two-dimensional Problems of Theory of Elasticity, M. Stroyizdat, Moscow, 1991.

Kuliyev, S. A.: Some problems of elasticity theory, Azerneshr, Baku, 2001.

Kuliyev, S. A.: Conformally-mapping Functions of Complex Domains, Lap Lambert Academic Publishing, Azerbaijan, Baku, 2017.

Muskhelishvili, N. I.: Some basic problems of mathematical theory of elasticity 2nd English edn., P. Noordhooff Ltd., the Netherlands, 1962.

Pan, Z., Cheng, Y., and Liu, J.: Stress analysis of a finite platewith a rectangular hole subjected to uniaxial tension using modified stress functions, Int. J. Mech. Sci, 75, 265-277, https://doi.org/10.1016/j.ijmecsci.2013.06.014, 2013.

Sharma, D. S.: Stress distribution around polygonal holes, Int. J. Mech. Sci., 65, 115-124, https://doi.org/10.1016/j.ijmecsci.2012.09.009, 2012.

Sharma, D. S.: Stress intensity factors for hypocycloidal hole with cusps in infinite anisotropic plate, Theo. Appl. Fract. Mech., 75, 44-52, https://doi.org/10.1016/j.tafmec.2014.10.005, 2015.

Sherman, D. I.: On a solution method of elasticity theory problems for doubly-connected domains, Dokl. AN SSSR, 55, 704-710, 1947.

Sherman, D. I.: On stresses in plane weight medium with two same, symmetrically arranged annular holes, PMM, 4, 751-761, 1951.

Tsurpal, I. A.: Experimental determination of elastic constants in the nonlinear theory of elasticity, Prikl. Mekh., 8, 55-63, 1962. 